\title{
Estimates of the potential kernel and Harnack's inequality for the anisotropic fractional Laplacian
}

\author{
by \\ Krzysztof Bogdan and PaWee Sztonyk (Wrocław)
}

\begin{abstract}
We characterize those homogeneous translation invariant symmetric nonlocal operators with positive maximum principle whose harmonic functions satisfy Harnack's inequality. We also estimate the corresponding semigroup and the potential kernel.
\end{abstract}

1. Main results and background. Let $\alpha \in(0,2)$ and $d \in\{1,2, \ldots\}$. We consider an arbitrary Lévy measure on $\mathbb{R}^{d} \backslash\{0\}$ which is symmetric, homogeneous: $\nu(r B)=r^{-\alpha} \nu(B)$, and nondegenerate (for definitions see Section 2). It yields a convolution semigroup of probability measures $\left\{P_{t}, t>0\right\}$ on $\mathbb{R}^{d}$. Each $P_{t}$ has a smooth density $p_{t}$. We consider the corresponding potential measure $\mathbb{V}=\int_{0}^{\infty} P_{t} d t$ and the potential kernel

$$
V(x)=\int_{0}^{\infty} p_{t}(x) d t, \quad x \in \mathbb{R}^{d} .
$$

$V(x)=|x|^{\alpha-d} V(x /|x|)$, but it may be infinite in some directions ([17, pp. $148-149])$. It is of interest to study continuity of $V$ on the unit sphere $\mathbb{S}$ in $\mathbb{R}^{d}$ under specific assumptions on $\nu$ (see (13)).

THEOREM 1. If $d>\alpha$ and $\nu$ is a $\gamma$-measure on $\mathbb{S}$ with $\gamma>d-2 \alpha$ then $V$ is continuous on $\mathbb{S}$.

The following partial converse shows that the threshold $d-2 \alpha$ is exact.

THEOREM 2. If $\mathbb{V}$ is a $\kappa$-measure on $\mathbb{S}$ then $\nu$ is a $(\kappa-2 \alpha)$-measure on $\mathbb{S}$.

In particular, if $V$ is bounded on $\mathbb{S}$ then $\nu$ is a $(d-2 \alpha)$-measure on $\mathbb{S}$.

2000 Mathematics Subject Classification: Primary 47D03, 31C05; Secondary 60J35, $60 \mathrm{G} 51$.

Key words and phrases: potential kernel, Harnack's inequality, relative Kato condition, Green function, stable process.

Research partially supported by KBN and RTN (HPRN-CT-2001-00273-HARP). 
We define an operator $\mathcal{A}$ on smooth functions $\varphi$ with compact support in $\mathbb{R}^{d}, \varphi \in C_{\mathrm{c}}^{\infty}\left(\mathbb{R}^{d}\right)$, by

$$
\begin{aligned}
\mathcal{A} \varphi(x) & =\int_{\mathbb{R}^{d}}\left(\varphi(x+y)-\varphi(x)-y \nabla \varphi(x) \mathbf{1}_{|y|<1}\right) \nu(d y) \\
& =\lim _{\varepsilon \rightarrow 0^{+}} \int_{|y|>\varepsilon}(\varphi(x+y)-\varphi(x)) \nu(d y) .
\end{aligned}
$$

$\mathcal{A}$ is a restriction of the infinitesimal generator of $\left\{P_{t}\right\}$ [35, Example 4.1.12], and what we refer to as the anisotropic fractional Laplacian in the title of the paper. In this connection we recall that in the special case of $\nu(d y)=$ $c|y|^{-d-\alpha} d y$ one obtains the fractional Laplacian $\Delta^{\alpha / 2}$. For properties of $\Delta^{\alpha / 2}$ and a discussion of equivalent definitions of its harmonic functions we refer the reader to [14].

Harmonic functions corresponding to $\mathcal{A}$, or $\nu$, are defined by the mean value property with respect to an appropriate family of harmonic measures (see Section 4). The main goal of the paper is to characterize those operators $\mathcal{A}$ for which Harnack's inequality holds, i.e., there is a constant $C=C(\alpha, \nu)$ such that for every function $u$ which is harmonic in the unit ball and nonnegative in $\mathbb{R}^{d}$,

$$
u\left(x_{1}\right) \leq C u\left(x_{2}\right), \quad\left|x_{1}\right|<1 / 2,\left|x_{2}\right|<1 / 2 .
$$

To this end we use the relative Kato condition (RK) meaning that there is a constant $K$ such that

$$
\int_{B(y, 1 / 2)}|y-v|^{\alpha-d} \nu(d v) \leq K \nu(B(y, 1 / 2)), \quad y \in \mathbb{R}^{d} .
$$

THEOREM 3. Harnack's inequality holds for $\mathcal{A}$ if and only if (RK) holds for $\nu$.

Theorem 3 is a strengthening of [17, Theorem 1], where an additional technical assumption was made: $\nu(d y) \leq c|y|^{-d-\alpha} d y$, to guarantee the boundedness of $V$ on $\mathbb{S}$. We now drop the assumption and the boundedness is obtained as the sole consequence of (2) via Theorem 1 . We also adapt some of our previous techniques from [17] to handle measures $\nu$ which are not absolutely continuous with respect to the Lebesgue measure on $\mathbb{R}^{d}$ (see, e.g., (27)).

Our estimates of the semigroup in Section 3 are based in part on ideas of [43], which concerns more complicated nonconvolutional semigroups. Another recent paper [53] gives involved estimates of our convolution semigroup $\left\{P_{t}\right\}$ in individual directions (see also [32] in this connection). Here we only need isotropic estimates of $\left\{P_{t}\right\}$ from above, and our considerations become simpler than those of [53] and [43]. 
In Sections 4-6 we develop the methods of [17]. That (2) implies (1) is proved by using a maximum principle for a Dynkin-type version of the operator $\mathcal{A}$ to explicitly estimate its Green function $G(x, v)$ for the unit ball (see Proposition 1 below). It is noteworthy that our proof of the estimate is specific to nonlocal operators, of which $\mathcal{A}$ is an illustrative special case. In particular it turns out that $G(x, v)$ has a singularity at the pole comparable to that of the Riesz kernel: $|v-x|^{\alpha-d}$. The singularity influences the magnitude of the corresponding Poisson kernel of the ball, $P(x, y)$, as given by the Ikeda-Watanabe formula (27). The influence is critical if and only if (2) fails to hold. This relates (2) to (1). Such a direct influence of the singularity of the potential kernel on the Poisson kernel does not occur for second order elliptic operators, which is why we can expect analogues of Theorem 3 only for nonlocal operators.

The recent development in the study of Harnack's inequality for general integro-differential operators similar to $\mathcal{A}$ was initiated in [6] (see also [16]). The class of operators considered gradually extended: see [47], [45], [5], [17], [2], and the references given there. We note that the operators dealt with in those papers are not translation invariant, nor are they homogeneous. On the other hand, the papers focus on sufficient conditions for Harnack's inequality and they are restricted by certain isotropic estimates of the operator's kernel from below.

Our confinement to translation invariant homogeneous operators $\mathcal{A}$ results in part from the fact that the problem of constructing the semigroup from a general nonlocal operator satisfying the positive maximum principle does not have a final solution yet. We refer the reader to [48, 49], [35, 36], [5], and [33]. A general survey of the subject and more references can be found in $[4,37,36]$. We refer the reader to $[29,3]$ for an account of the related potential theory of second order elliptic operators. We point out that while a symmetric second order elliptic operator with constant coefficients is merely a linear transformation of the Laplacian, the operators $\mathcal{A}$ and their harmonic functions considered here are very diverse ([17]).

The remainder of the paper is organized as follows. The first definitions are given in Section 2. In Section 3 we estimate the semigroup (see (17) below) and the potential measure $\mathbb{V}$ and we prove our first two theorems. In Section 4 we give preliminaries needed for the proof of Theorem 3, which is presented in Sections 5 and 6. In Section 6 we also recall after [17] two explicit examples to show how irregular the Lévy measure $\nu$ can be for Harnack's inequality to hold or to fail for $\mathcal{A}$.

At the end of the paper we mention some remaining open problems. 
2. Preliminaries. For $x \in \mathbb{R}^{d}$ and $r>0$ we let $|x|=\sqrt{\sum_{i=1}^{d} x_{i}^{2}}$ and $B(x, r)=\left\{y \in \mathbb{R}^{d}:|y-x|<r\right\}$. We define $\mathbb{S}=\left\{x \in \mathbb{R}^{d}:|x|=1\right\}$. All the sets, functions and measures considered below will be Borel. For a measure $\lambda$ on $\mathbb{R}^{d},|\lambda|$ denotes its total mass. For a function $f$ we let $\lambda(f)=\int f d \lambda$ whenever the integral makes sense. When $|\lambda|<\infty$ and $n=1,2, \ldots$ we let $\lambda^{n}$ denote the $n$-fold convolution of $\lambda$ with itself:

$$
\lambda^{n}(f)=\int f\left(x_{1}+\cdots+x_{n}\right) \lambda\left(d x_{1}\right) \cdots \lambda\left(d x_{n}\right) .
$$

We also let $\lambda^{0}=\delta_{0}$, the evaluation at 0 . We call $\lambda$ degenerate if there is a proper linear subspace $M$ of $\mathbb{R}^{d}$ such that $\operatorname{supp}(\lambda) \subset M$; otherwise we call $\lambda$ nondegenerate.

In what follows we consider measures $\mu$ concentrated on $\mathbb{S}$. We assume that $\mu$ is positive, finite, nondegenerate (in particular $\mu \neq 0$ ), and symmetric:

$$
\mu(D)=\mu(-D), \quad D \subset \mathbb{R}^{d} .
$$

We call $\mu$ a spectral measure. We let

$$
\nu(D)=\int_{\mathbb{S}} \int_{0}^{\infty} \mathbf{1}_{D}(r \xi) r^{-1-\alpha} d r \mu(d \xi), \quad D \subset \mathbb{R}^{d},
$$

where $\mathbf{1}_{D}$ is the indicator function of $D$. Note that $\nu$ is symmetric. It is a Lévy measure on $\mathbb{R}^{d}$, i.e.

$$
\int_{\mathbb{R}^{d}} \min \left(|y|^{2}, 1\right) \nu(d y)<\infty
$$

For $r>0$ and a function $\varphi$ on $\mathbb{R}^{d}$ we consider its dilation $\varphi_{r}(y)=\varphi(y / r)$, and we note that $\nu\left(\varphi_{r}\right)=r^{-\alpha} \nu(\varphi)$. In particular $\nu$ is homogeneous: $\nu(r B)=$ $r^{-\alpha} \nu(B)$ for $B \subset \mathbb{R}^{d}$. Similarly, if $\varphi \in C_{\mathrm{c}}^{\infty}\left(\mathbb{R}^{d}\right)$, then $\mathcal{A}\left(\varphi_{r}\right)=r^{-\alpha}(\mathcal{A} \varphi)_{r}$. This is the homogeneity of $\mathcal{A}$. In connection with the rest of our statement in the abstract we recall that every operator $A$ on $C_{\mathrm{c}}^{\infty}\left(\mathbb{R}^{d}\right)$ which satisfies the positive maximum principle:

$$
\sup _{y \in \mathbb{R}^{d}} \varphi(y)=\varphi(x) \geq 0 \quad \text { implies } \quad A \varphi(x) \leq 0,
$$

is given uniquely in the form

$$
\begin{aligned}
A \varphi(x)= & \sum_{i, j=1}^{d} a_{i j}(x) D_{x_{i}} D_{x_{j}} \varphi(x)+b(x) \nabla \varphi(x)-c(x) \varphi(x) \\
& +\int_{\mathbb{R}^{d}}\left(\varphi(x+y)-\varphi(x)-y \nabla \varphi(x) \mathbf{1}_{|y|<1}\right) \nu(x, d y) .
\end{aligned}
$$

Here $y \nabla \varphi$ is the scalar product of $y$ and the gradient of $\varphi$ and, for every $x$, $a(x)=\left(a_{i j}(x)\right)_{i, j=1}^{n}$ is a nonnegative definite real symmetric matrix, the vector $b(x)=\left(b_{i}(x)\right)_{i=1}^{d}$ has real coordinates, $c(x) \geq 0$, and $\nu(x, \cdot)$ is a Lévy 
measure. This description is due to Courrège (see [33, Proposition 2.10], [49, Chapter 2] or [35, Chapter 4.5]). For translation invariant operators $A$ the characteristics $a, b, c$, and $\nu$ are independent of $x$. If $A$ is symmetric:

$$
\int_{\mathbb{R}^{d}} A \varphi(x) \phi(x) d x=\int_{\mathbb{R}^{d}} A \phi(x) \varphi(x) d x \quad \text { for } \varphi, \phi \in C_{\mathrm{c}}^{\infty}\left(\mathbb{R}^{d}\right),
$$

then $b=0$ and $\nu$ is necessarily symmetric (see, e.g., [35, p. 251] and [33, Corollary 2.14]). If $A$ is homogeneous but not local ([35]) then $a=0$ and $\nu$ must be homogeneous, hence (3) holds with some $\alpha \in(0,2)$ (note that $\mathcal{A} \varphi(0)=\nu(\varphi)$ if $\left.\varphi \in C_{\mathrm{c}}^{\infty}\left(\mathbb{R}^{d} \backslash\{0\}\right)\right)$.

We now construct the corresponding semigroup (for a more axiomatic introduction to convolution semigroups we refer the reader to [7, 35]). For $\varepsilon>0$ we let $\widehat{\nu}_{\varepsilon}=\mathbf{1}_{B(0, \varepsilon)^{\mathrm{c}}} \nu$, i.e. $\widehat{\nu}_{\varepsilon}(f)=\nu\left(\mathbf{1}_{B(0, \varepsilon)^{\mathrm{c}}} f\right)$, and we let $\widetilde{\nu}_{\varepsilon}=\mathbf{1}_{B(0, \varepsilon)} \nu$. We consider the probability measures

$$
\begin{aligned}
\widehat{P}_{t}^{\varepsilon} & =\exp \left(t\left(\widehat{\nu}_{\varepsilon}-\left|\widehat{\nu}_{\varepsilon}\right| \delta_{0}\right)\right)=\sum_{n=0}^{\infty} \frac{\left.t^{n}\left(\widehat{\nu}_{\varepsilon}-\left|\widehat{\nu}_{\varepsilon}\right| \delta_{0}\right)\right)^{n}}{n !} \\
& =e^{-t\left|\widehat{\nu}_{\varepsilon}\right|} \sum_{n=0}^{\infty} \frac{t^{n} \widehat{\nu}_{\varepsilon}^{n}}{n !}, \quad t>0 .
\end{aligned}
$$

Here $\widehat{\nu}_{\varepsilon}^{n}=\left(\widehat{\nu}_{\varepsilon}\right)^{n}$. The $\widehat{P}_{t}^{\varepsilon}$ form a convolution semigroup:

$$
\widehat{P}_{t}^{\varepsilon} * \widehat{P}_{s}^{\varepsilon}=\widehat{P}_{s+t}^{\varepsilon}, \quad s, t>0 .
$$

The Fourier transform of $\widehat{P}_{t}^{\varepsilon}$ is

$$
\mathcal{F}\left(\widehat{P}_{t}^{\varepsilon}\right)(u)=\int e^{i u y} \widehat{P}_{t}^{\varepsilon}(d y)=\exp \left(t \int\left(e^{i u y}-1\right) \widehat{\nu}_{\varepsilon}(d y)\right), \quad u \in \mathbb{R}^{d} .
$$

The measures $\widehat{P}_{t}^{\varepsilon}$ weakly converge to a probability measure $P_{t}$ as $\varepsilon \rightarrow 0$ (this essentially depends on (6) below). $\left\{P_{t}, t>0\right\}$ is also a convolution semigroup and $\mathcal{F}\left(P_{t}\right)(u)=\exp (-t \Phi(u))$, where

$$
\begin{aligned}
\Phi(u) & =-\int\left(e^{i u y}-1-i u y \mathbf{1}_{B(0,1)}(y)\right) \nu(d y) \\
& =-\int(\cos (u y)-1) \nu(d y)=\frac{\pi}{2 \sin (\pi \alpha / 2) \Gamma(1+\alpha)} \int_{\mathbb{S}}|u \xi|^{\alpha} \mu(d \xi) .
\end{aligned}
$$

Since $\mu$ is finite and nondegenerate,

$$
\Phi(u)=|u|^{\alpha} \Phi(u /|u|) \approx|u|^{\alpha} .
$$

We call $\nu$ the Lévy measure of the semigroup $\left\{P_{t}, t \geq 0\right\}[33,7]$.

By a similar limiting procedure we construct the semigroup $\left\{\widetilde{P}_{t}^{\varepsilon}, t>0\right\}$ such that

$$
\mathcal{F}\left(\widetilde{P}_{t}^{\varepsilon}\right)(u)=\exp \left(t \int\left(e^{i u y}-1-i u y \mathbf{1}_{B(0,1)}(y)\right) \widetilde{\nu}_{\varepsilon}(d y)\right) .
$$


Note that

$$
\int_{\mathbb{R}^{d}}|y|^{2} \widetilde{P}_{t}^{\varepsilon}(d y)=t \int_{\mathbb{R}^{d}}|y|^{2} \widetilde{\nu}_{\varepsilon}(d y) .
$$

The Lévy measures of $\left\{\widetilde{P}_{t}^{\varepsilon}\right\}$ and $\left\{\widehat{P}_{t}^{\varepsilon}\right\}$ are $\widetilde{\nu}_{\varepsilon}$ and $\widehat{\nu}_{\varepsilon}$, respectively, and we have

$$
P_{t}=\widetilde{P}_{t}^{\varepsilon} * \widehat{P}_{t}^{\varepsilon}
$$

The measures $P_{t}$ and $\widetilde{P}_{t}^{\varepsilon}$ have rapidly decreasing Fourier transform, hence they are absolutely continuous with bounded smooth densities denoted $p_{t}(x)$ and $\widetilde{p}_{t}^{\varepsilon}(x)$, respectively. Of course,

$$
p_{t}=\widetilde{p}_{t}^{\varepsilon} * \widehat{P}_{t}^{\varepsilon} .
$$

By using (5) we obtain the scaling property of $\left\{p_{t}\right\}$ :

$$
p_{t}(x)=t^{-d / \alpha} p_{1}\left(t^{-1 / \alpha} x\right), \quad x \in \mathbb{R}^{d} .
$$

In particular,

$$
p_{t}(x) \leq c t^{-d / \alpha} \text {. }
$$

We define the potential measure of the semigroup $\left\{P_{t}\right\}$ :

$$
\mathbb{V}(D)=\int_{0}^{\infty} P_{t}(D) d t, \quad D \subset \mathbb{R}^{d} .
$$

By (10), $\mathbb{V}$ is finite on bounded subsets of $\mathbb{R}^{d}$ if $d>\alpha$. Let

$$
V(x)=\int_{0}^{\infty} p_{t}(x) d t, \quad x \in \mathbb{R}^{d},
$$

so that

$$
\mathbb{V}(D)=\int_{D} V(x) d x, \quad D \subset \mathbb{R}^{d} .
$$

We call $V(x)$ the potential kernel of the stable semigroup. By (9),

$$
V(x)=|x|^{\alpha-d} V(x /|x|), \quad x \neq 0,
$$

and $\mathbb{V}(r D)=r^{\alpha} \mathbb{V}(D)$ for $r>0, D \subset \mathbb{R}^{d}$.

If $d=1$ then up to a constant there is only one measure $\nu$ to consider: $\nu(d y)=|y|^{-1-\alpha} d y$, corresponding to $\mathcal{A}=c \Delta^{\alpha / 2}$. This case of $d=1$ is not excluded from our considerations but it is sometimes trivial. In particular, if $d=1 \leq \alpha$ then $V \equiv \infty$ ([7, Example 14.30]). We refer to [18] for more information and references on the case $d=1 \leq \alpha$.

Constants in this paper mean positive real numbers. We often write $f \approx g$ to indicate that there is $c=c(\alpha, \mu)$, i.e. a constant $c$ depending only on $\alpha$ and $\mu$, such that $c^{-1} f \leq g \leq c f$. 
3. Estimates of the semigroup and potential measure. A general reference to the potential theory of convolution semigroups is [7] (see also $[35,36])$.

We consider an auxiliary scale of smoothness for $\nu$.

DeFinition 1. We say that $\nu$ is a $\gamma$-measure on $\mathbb{S}$ if

$$
\nu(B(x, r)) \leq c r^{\gamma}, \quad|x|=1,0<r<1 / 2 .
$$

Since $\nu(d r d \theta)=r^{-1-\alpha} d r \mu(d \theta)$, it is at least a 1-measure and at most a $d$-measure on $\mathbb{S}$. If $\nu$ is a $\gamma$-measure with $\gamma>1$, then $\mu$ has no atoms. Moreover $\nu$ is a $d$-measure if and only if it is absolutely continuous with respect to the Lebesgue measure and has a density function which is locally bounded on $\mathbb{R}^{d} \backslash\{0\}$. We refer the reader to [26] and [30] for considerations relating to this case.

In the remainder of this section we fix $1 \leq \gamma \leq d$ and we assume that $\nu$ is a $\gamma$-measure on $\mathbb{S}$.

We first estimate individual terms in the series of (4).

Lemma 1. There exists $C=C(\alpha, \mu)$ such that for $\varepsilon>0$ and $n=1,2, \ldots$ we have

$$
\widehat{\nu}_{\varepsilon}^{n}(B(x, r)) \leq C^{n} r^{\gamma} \varepsilon^{-(n-1) \alpha}, \quad|x|=1,
$$

provided $0<r<\max \left(\varepsilon / 3,1 / 5^{n}\right)$.

Proof. We proceed by induction. Note that (14) holds for $n=1$ by (13). Let $c_{0}$ and $n$ be such that (14) is satisfied with $C=c_{0}$. We first assume that $r<\varepsilon / 3$. For every $x \in \mathbb{S}$ by homogeneity of $\nu$ and (13) we have

$$
\begin{aligned}
\widehat{\nu}_{\varepsilon}^{n+1}(B(x, r)) & =\int_{|x-y|>2 \varepsilon / 3} \widehat{\nu}_{\varepsilon}(B(x-y, r)) \widehat{\nu}_{\varepsilon}^{n}(d y) \\
& \leq \int_{|x-y|>2 \varepsilon / 3} \nu(B(x-y, r)) \widehat{\nu}_{\varepsilon}^{n}(d y) \\
& =\int_{|x-y|>2 \varepsilon / 3}|x-y|^{-\alpha} \nu\left(B\left(\frac{x-y}{|x-y|}, \frac{r}{|x-y|}\right)\right) \widehat{\nu}_{\varepsilon}^{n}(d y) \\
& \leq c_{1} r^{\gamma} \int_{|x-y|>2 \varepsilon / 3}|x-y|^{-\alpha-\gamma} \widehat{\nu}_{\varepsilon}^{n}(d y)
\end{aligned}
$$

(note that $r /|x-y|<1 / 2$ provided $|x-y|>2 \varepsilon / 3$ ). Now let $\varepsilon / 3 \leq r<$ $1 / 5^{n+1}$. Then $2 r+\varepsilon<1 / 5^{n}$ and by induction

$$
\begin{aligned}
\int_{|x-y|<2 r+\varepsilon} \widehat{\nu}_{\varepsilon}(B(x-y, r)) \widehat{\nu}_{\varepsilon}^{n}(d y) & \leq\left|\widehat{\nu}_{\varepsilon}\right| \widehat{\nu}_{\varepsilon}^{n}(B(x, 2 r+\varepsilon)) \\
& \leq \frac{|\mu|}{\alpha} \varepsilon^{-\alpha} c_{0}^{n}(2 r+\varepsilon)^{\gamma} \varepsilon^{-(n-1) \alpha} \leq c_{0}^{n} c_{2} r^{\gamma} \varepsilon^{-n \alpha}
\end{aligned}
$$


for some $c_{2}=c_{2}(\alpha, \mu)$; and by homogeneity of $\nu$ and (13) we get

$$
\begin{aligned}
& \int_{|x-y|>2 r+\varepsilon} \widehat{\nu}_{\varepsilon}(B(x-y, r)) \widehat{\nu}_{\varepsilon}^{n}(d y) \leq \int_{|x-y|>2 r+\varepsilon} \nu(B(x-y, r)) \widehat{\nu}_{\varepsilon}^{n}(d y) \\
& \quad \leq \int_{|x-y|>2 r+\varepsilon} c_{1} r^{\gamma}|x-y|^{-\alpha-\gamma} \widehat{\nu}_{\varepsilon}^{n}(d y) \leq c_{1} r^{\gamma} \int_{|x-y|>2 \varepsilon / 3}|x-y|^{-\alpha-\gamma} \widehat{\nu}_{\varepsilon}^{n}(d y) .
\end{aligned}
$$

From the above we have

$$
\widehat{\nu}_{\varepsilon}^{n+1}(B(x, r)) \leq c_{1} r^{\gamma} \int_{|x-y|>2 \varepsilon / 3}|x-y|^{-\alpha-\gamma} \widehat{\nu}_{\varepsilon}^{n}(d y)+c_{0}^{n} c_{2} r^{\gamma} \varepsilon^{-n \alpha}
$$

for all $0<r<\max \left(\varepsilon / 3,1 / 5^{n+1}\right)$.

Let $L_{\varepsilon}=\left\lfloor\log _{5}(3 / 2 \varepsilon)\right\rfloor$. If $2 \varepsilon / 3<1 / 5^{n}$ then we get by induction

$$
\begin{aligned}
& \int_{2 \varepsilon / 3<|x-y|<1 / 5^{n}}|x-y|^{-\alpha-\gamma} \widehat{\nu}_{\varepsilon}^{n}(d y) \\
\leq & \sum_{k=n}^{L_{\varepsilon}} \int_{1 / 5^{k+1}<|x-y|<1 / 5^{k}}|x-y|^{-\alpha-\gamma} \widehat{\nu}_{\varepsilon}^{n}(d y) \leq \sum_{k=n}^{L_{\varepsilon}}\left(5^{k+1}\right)^{\alpha+\gamma} \widehat{\nu}_{\varepsilon}^{n}\left(B\left(x, 1 / 5^{k}\right)\right) \\
\leq & c_{0}^{n} 5^{\alpha+\gamma} \varepsilon^{-(n-1) \alpha} \sum_{k=1}^{L_{\varepsilon}} 5^{k \alpha} \leq c_{0}^{n} c_{3} \varepsilon^{-n \alpha},
\end{aligned}
$$

where $c_{3}=c_{3}(\alpha, \mu)$. Also,

$$
\int_{|x-y|>1 / 5^{n}}|x-y|^{-\alpha-\gamma} \widehat{\nu}_{\varepsilon}^{n}(d y) \leq\left(5^{\alpha+\gamma}\right)^{n}\left|\widehat{\nu}_{\varepsilon}^{n}\right|=\left(5^{\alpha+\gamma}|\mu| / \alpha\right)^{n} \varepsilon^{-n \alpha} \leq c_{0}^{n} \varepsilon^{-n \alpha},
$$

by taking large $c_{0}$. We get

$$
\int_{|x-y|>2 \varepsilon / 3}|x-y|^{-\alpha-\gamma} \widehat{\nu}_{\varepsilon}^{n}(d y) \leq c_{0}^{n} \varepsilon^{-n \alpha}\left(c_{3}+1\right),
$$

and (15) yields

$$
\widehat{\nu}_{\varepsilon}^{n+1}(B(x, r)) \leq c_{0}^{n+1} r^{\gamma} \varepsilon^{-n \alpha} .
$$

Corollary 2. There exists $C=C(\alpha, \mu)$ such that

$$
\widehat{\nu}_{\varepsilon}^{n}(B(x, \lambda \varepsilon)) \leq C^{n} \lambda^{\gamma}\left(1+\lambda^{\alpha}\right) \varepsilon^{\gamma-(n-1) \alpha}, \quad \lambda>0, \varepsilon>0,|x|=1 .
$$

Proof. Lemma 1 yields (16) for $\lambda \varepsilon<1 / 5^{n}$. For $\lambda \varepsilon \geq 1 / 5^{n}$ we have

$$
\widehat{\nu}_{\varepsilon}^{n}(B(x, \lambda \varepsilon)) \leq\left|\widehat{\nu}_{\varepsilon}^{n}\right|=\frac{|\mu|^{n}}{\alpha^{n}} \varepsilon^{-n \alpha} \leq\left(\frac{|\mu|}{\alpha} 5^{\alpha+\gamma}\right)^{n} \lambda^{\alpha+\gamma} \varepsilon^{\gamma-(n-1) \alpha} .
$$

In what follows we write $\widehat{P}_{t}=\widehat{P}_{t}^{t^{1 / \alpha}}$ and $\widetilde{P}_{t}=\widetilde{P}_{t}^{t^{1 / \alpha}}$.

Corollary 3. There exists $C=C(\alpha, \mu)$ such that

$$
\widehat{P}_{t}\left(B\left(x, \lambda t^{1 / \alpha}\right)\right) \leq C \lambda^{\gamma}\left(1+\lambda^{\alpha}\right) t^{1+\gamma / \alpha}, \quad \lambda>0, t>0,|x|=1 .
$$


Proof. Corollary 2 yields

$$
\begin{aligned}
\widehat{P}_{t}\left(B\left(x, \lambda t^{1 / \alpha}\right)\right) & =e^{-|\mu| / \alpha} \sum_{n=0}^{\infty} \frac{t^{n} \widehat{\nu}_{t^{1 / \alpha}}^{n}\left(B\left(x, \lambda t^{1 / \alpha}\right)\right)}{n !} \\
\leq & e^{-|\mu| / \alpha} \sum_{n=0}^{\infty} \frac{c^{n} \lambda^{\gamma}\left(1+\lambda^{\alpha}\right) t^{1+\gamma / \alpha}}{n !}=e^{c-|\mu| / \alpha} \lambda^{\gamma}\left(1+\lambda^{\alpha}\right) t^{1+\gamma / \alpha}
\end{aligned}
$$

Corollary 4. $\widehat{P}_{1}(B(y, \lambda)) \leq C \lambda^{\gamma}\left(1+\lambda^{\alpha}\right)|y|^{-\alpha-\gamma}$ for $y \in \mathbb{R}^{d}$ and $\lambda>0$.

Proof. Let $y \in \mathbb{R}^{d} \backslash\{0\}$ and $x=y /|y|, t=|y|^{-\alpha}$. By scaling and Corollary 3 we have

$$
\widehat{P}_{1}(B(y, \lambda))=\widehat{P}_{t}\left(B\left(x, \lambda t^{1 / \alpha}\right)\right) \leq c \lambda^{\gamma}\left(1+\lambda^{\alpha}\right)|y|^{-\alpha-\gamma} .
$$

We note that for every $q>0$ we have $\int|y|^{q} \widetilde{P}_{1}(d y)<\infty$, because the support of $\widetilde{\nu}_{1}$ is bounded ([46]). A simple reasoning based on this and the boundedness of the derivative of $\widetilde{p}_{1}$ yields

$$
\widetilde{p}_{1}(y) \leq c_{q}(1+|y|)^{-q}, \quad q>0, y \in \mathbb{R}^{d}
$$

(see [43, Lemma 9]).

Lemma 5. For every $q>0$ there exists $C=C(\alpha, \mu, q)$ such that

$$
\widetilde{P}_{1}(B(z, \varrho)) \leq C(1+|z|)^{-q} \varrho^{d}, \quad \varrho \leq 1, z \in \mathbb{R}^{d} .
$$

Proof. If $|z|<2$ then $\widetilde{P}_{1}(B(z, \varrho))=\int_{B(z, \varrho)} \widetilde{p}_{1}(y) d y \leq c \varrho^{d} \leq c(1+|z|)^{-q} \varrho^{d}$. If $|z| \geq 2$ then $\widetilde{P}_{1}(B(z, \varrho)) \leq c(1+|z| / 2)^{-q} \varrho^{d} \leq c(1+|z|)^{-q} \varrho^{d}$.

The proof of the following lemma is a simplification of the proof of [43, Theorem 3].

Lemma 6. $P_{1}(B(z, \varrho)) \leq C|z|^{-\alpha-\gamma} \varrho^{d}$ for $z \in \mathbb{R}^{d}$ and $0<\varrho \leq 1$.

Proof. By (7), Lemma 5, and Corollary 4,

$$
\begin{aligned}
P_{1}(B(z, \varrho)) & =\widetilde{P}_{1} * \widehat{P}_{1}(B(z, \varrho))=\int_{\mathbb{R}^{d}} \widetilde{P}_{1}(B(z-y, \varrho)) \widehat{P}_{1}(d y) \\
& =\int_{0}^{1} \widehat{P}_{1}\left(\left\{y: \widetilde{P}_{1}(B(z-y, \varrho))>s\right\}\right) d s \\
& \leq \int_{0}^{1} \widehat{P}_{1}\left(\left\{y: c(1+|z-y|)^{-q} \varrho^{d}>s\right\}\right) d s \\
& \leq \int_{0}^{c \varrho^{d}} \widehat{P}_{1}\left(B\left(z, c^{1 / q} s^{-1 / q} \varrho^{d / q}\right)\right) d s
\end{aligned}
$$




$$
\begin{aligned}
& \leq c \int_{0}^{c \varrho^{d}}\left(c^{1 / q} s^{-1 / q} \varrho^{d / q}\right)^{\gamma}\left(1+\left(c^{1 / q} s^{-1 / q} \varrho^{d / q}\right)^{\alpha}\right)|z|^{-\gamma-\alpha} d s \\
& =c|z|^{-\gamma-\alpha}\left[\varrho^{d \gamma / q} \int_{0}^{c \varrho^{d}} s^{-\gamma / q} d s+\varrho^{d(\gamma+\alpha) / q} \int_{0}^{c \varrho^{d}} s^{-(\gamma+\alpha) / q} d s\right] \\
& =c|z|^{-\gamma-\alpha}\left[\varrho^{d \gamma / q}\left(\varrho^{d}\right)^{1-\gamma / q}+\varrho^{d(\gamma+\alpha) / q}\left(\varrho^{d}\right)^{1-(\gamma+\alpha) / q}\right]=c|z|^{-\gamma-\alpha} \varrho^{d} .
\end{aligned}
$$

The following two corollaries are our main estimates of the semigroup. Corollary 8 is an analogue of [43, Theorem 3], while (17) corresponds to [53].

Corollary 7. $P_{1}(B(z, \varrho)) \leq C(1+|z|)^{-\alpha-\gamma} \varrho^{d} \quad$ if $0 \leq \varrho<|z| / 2$.

Proof. We recall that $p_{1}(y)=P_{1}(d y) / d y$ is bounded and so Lemma 6 yields

$$
p_{1}(y) \leq c(1+|y|)^{-\gamma-\alpha}, \quad y \in \mathbb{R}^{d} .
$$

If $0 \leq \varrho<|z| / 2$ then

$$
P_{1}(B(z, \varrho)) \leq c \int_{B(z, \varrho)}(1+|y|)^{-\gamma-\alpha} d y \leq(1+|z|)^{-\alpha-\gamma} \varrho^{d}
$$

Corollary 8. $P_{t}(B(x, \varrho)) \leq C t^{1+(\gamma-d) / \alpha} \varrho^{d}$ provided $|x|=1, t>0$, and $0 \leq \varrho \leq t^{1 / \alpha}$.

Proof. By scaling and Lemma 6 we have

$$
P_{t}(B(x, \varrho))=P_{1}\left(B\left(x t^{-1 / \alpha}, \varrho t^{-1 / \alpha}\right)\right) \leq c t^{1+(\gamma-d) / \alpha} \varrho^{d} .
$$

Proof of Theorem 1. Let $|x|=1,0 \leq \varrho<1 / 2$. By scaling and Corollary 7 ,

$$
\begin{aligned}
\mathbb{V}(B(x, \varrho)) & =\int_{0}^{\infty} P_{t}(B(x, \varrho)) d t=\int_{0}^{\infty} P_{1}\left(B\left(x t^{-1 / \alpha}, \varrho t^{-1 / \alpha}\right)\right) d t \\
& \leq c \varrho^{d} \int_{0}^{\infty}\left(1+t^{-1 / \alpha}\right)^{-\gamma-\alpha} t^{-d / \alpha} d t
\end{aligned}
$$

The integral is finite because $-d / \alpha<-1$ and $(\gamma+\alpha-d) / \alpha>-1$. Let $y \in \mathbb{R}^{d} \backslash\{0\}, x=y /|y|$. By scaling, a change of variable, and (17),

$$
\begin{aligned}
V(y) & =\int_{0}^{\infty} t^{-d / \alpha} p_{1}\left(y t^{-1 / \alpha}\right) d t=|y|^{\alpha-d} \int_{0}^{\infty} t^{-d / \alpha} p_{1}\left(x t^{-1 / \alpha}\right) d t \\
& \leq|y|^{\alpha-d} \int_{0}^{\infty} t^{-d / \alpha}\left(1+t^{-1 / \alpha}\right)^{-\gamma-\alpha} d t \leq c|y|^{\alpha-d} .
\end{aligned}
$$

The first integral above is locally uniformly convergent on $\mathbb{R}^{d} \backslash\{0\}$, hence $V$ is continuous there. 
We now proceed to our converse, Theorem 2. We propose a general approach based on a simple study of the generator $\mathcal{A}$. We first note that

$$
p_{t}(x)>0, \quad x \in \mathbb{R}^{d} \quad(t>0)
$$

(see [52] or [43, Lemma 5]). In fact, (18) easily follows from (8), (4), continuity of $\widetilde{p}_{t}^{\varepsilon}$, and the fact that $\operatorname{supp}(\nu)+\cdots+\operatorname{supp}(\nu)(d$ times $)$ equals $\mathbb{R}^{d}$.

By (18), (12), and continuity of $p_{t}$ for $t>0$, there is a constant $c=c(\alpha, \mu)$ such that

$$
V(x) \geq c|x|^{\alpha-d}, \quad x \in \mathbb{R}^{d} .
$$

Lemma 9. Let $d>\alpha$. For all $\varphi \in C_{\mathrm{c}}^{\infty}\left(\mathbb{R}^{d}\right)$ we have

$$
\int_{\mathbb{R}^{d}} \mathcal{A} \varphi(x-y) \mathbb{V}(d y)=-\varphi(x), \quad x \in \mathbb{R}^{d},
$$

where the integral is absolutely convergent.

This is well known (see, e.g., [36, Theorem 3.5.78]). We only note that $|\mathcal{A} \varphi(x)| \leq c(1+|x|)^{-1-\alpha}$. The absolute convergence follows from this and the homogeneity of $\mathbb{V}$.

Proof of Theorem 2. If $d=1 \leq \alpha$ then $V \equiv \infty$ and there is nothing to prove. Thus we assume that $d>\alpha$. We fix a function $\phi \in C_{\mathrm{c}}^{\infty}\left(\mathbb{R}^{d}\right)$ such that $\phi \geq 0, \operatorname{supp} \phi \subset B(0,1 / 2)$ and $\phi=1$ on $B(0,1 / 3)$. Let $r>0$. Put $\phi_{r}(x)=$ $\phi(x / r)$ and $\Lambda_{r}(x)=\mathcal{A} \phi_{r}(x)$. Homogeneity of $\mathcal{A}$ yields $\Lambda_{r}(x)=r^{-\alpha} \Lambda_{1}(x / r)$. Note that $\mathcal{A} \phi=\Lambda_{1}$ is bounded, hence there is a constant $c$ such that

$$
\Lambda_{r}(x) \geq-c r^{-\alpha} \text {. }
$$

If $|x| \geq r / 2$ then $\Lambda_{r}(x) \geq 0$, and in fact $\Lambda_{r}(x) \geq \nu(B(x, r / 3))$. Let $|x|>r$. From Lemma 9 we have

$$
\begin{aligned}
0 & =\int_{\mathbb{R}^{d}} \Lambda_{r}(x-y) \mathbb{V}(d y) \geq \int_{B(x, r / 2)} \Lambda_{r}(x-y) \mathbb{V}(d y)+\int_{B(0, r / 4)} \Lambda_{r}(x-y) \mathbb{V}(d y) \\
& \geq-c r^{-\alpha} \mathbb{V}\left((B(x, r / 2))+\int_{B(0, r / 4)} \nu(B(x-y, r / 3)) \mathbb{V}(d y)\right. \\
& \geq-c r^{-\alpha} \mathbb{V}((B(x, r / 2))+\mathbb{V}(B(0, r / 4)) \nu(B(x, r / 12))
\end{aligned}
$$

Since $\mathbb{V}(B(0, r / 4))=r^{\alpha} \mathbb{V}(B(0,1 / 4))$ and $\mathbb{V}(B(0,1 / 4))<\infty$ we get

$$
\nu(B(x, r / 12)) \leq c r^{-2 \alpha} \mathbb{V}((B(x, r / 2)), \quad|x|>r .
$$

We note that similar results can also be derived from the lower bounds for the semigroup as given in [53, Theorem 1.1].

4. Harnack's inequality: preliminaries. The general references for this section are [22, 23], [46], [8], or [10]. The Lévy measure $\nu$ yields a standard symmetric stable Lévy process $\left(X_{t}, P^{x}\right)$ with generating triplet $(0, \nu, 0)$. Namely, the transition probabilities of the process $\left(X_{t}, P^{x}\right)$ are 
$P(t, x, A)=P_{t}(A-x), t>0, x \in \mathbb{R}^{d}, A \subset \mathbb{R}^{d}$, and $P(0, x, A)=\mathbf{1}_{A}(x)$, where $\left\{P_{t}, t \geq 0\right\}$ is the stable semigroup of measures introduced in Preliminaries. The process is strong Markov with respect to the so-called standard filtration.

The process conveniently leads to a definition of harmonic measures $\omega_{D}^{x}$, and their properties (21) and (24) below. For an analytic definition of these, called the fundamental family, we refer to [7] (see also [40, 9]).

For open $U \subset \mathbb{R}^{d}$ we define $\tau_{U}=\inf \left\{t \geq 0: X_{t} \notin U\right\}$, the first exit time of $U$. We write $\omega_{D}^{x}$ for the harmonic measure of (open) $D$ :

$$
\omega_{D}^{x}(A)=P^{x}\left(\tau_{D}<\infty, X_{\tau_{D}} \in A\right), \quad x \in \mathbb{R}^{d}, A \subset \mathbb{R}^{d} .
$$

By the strong Markov property

$$
\omega_{D}^{x}(A)=\int \omega_{D}^{y}(A) \omega_{U}^{x}(d y) \quad \text { if } U \subset D .
$$

We say that a function $u$ on $\mathbb{R}^{d}$ is harmonic in open $D \subset \mathbb{R}^{d}$ if

$$
u(x)=E^{x} u\left(X_{\tau_{U}}\right)=\int_{U^{\mathrm{c}}} u(y) \omega_{U}^{x}(d y), \quad x \in \mathbb{R}^{d},
$$

for every bounded open set $U$ with the closure $\bar{U}$ contained in $D$. It is called regular harmonic in $D$ if (22) holds for $U=D$. If $D$ is unbounded then $E^{x} u\left(X_{\tau_{D}}\right)=E^{x}\left[\tau_{D}<\infty ; u\left(X_{\tau_{D}}\right)\right]$ by convention. Under (22) it will only be assumed that the expectation in (22) is well defined (but not necessarily finite). Regular harmonicity implies harmonicity, and it is inherited by subsets $U \subset D$. This follows from (21).

We denote by $p_{t}^{D}(x, v)$ the transition density of the process killed at the first exit from $D$ :

$$
p_{t}^{D}(x, v)=p(t, x, v)-E^{x}\left[\tau_{D}<t ; p\left(t-\tau_{D}, X_{\tau_{D}}, v\right)\right], \quad t>0, x, v \in \mathbb{R}^{d} .
$$

Here $p(t, x, v)=p_{t}(v-x)$. For convenience we will assume that $D$ is regular: $P^{x}\left[\inf \left\{t>0: X_{t} \notin D\right\}=0\right]=1$ for $x \in D^{\mathrm{c}}$ (see [23, 22]). Then $p_{t}^{D}$ is symmetric: $p_{t}^{D}(x, v)=p_{t}^{D}(v, x), x, v \in D$ (see, e.g., [24]). The strong Markov property yields

$$
p(t, x, v)=E^{x}\left[p\left(t-\tau_{D}, X_{\tau_{D}}, v\right) ; \tau_{D}<t\right], \quad x \in D, v \in D^{\mathrm{c}} .
$$

In particular, $p_{t}^{D}(x, v)=0$ if $x \in D, v \in D^{\mathrm{c}}$. We let

$$
G_{D}(x, v)=\int_{0}^{\infty} p_{t}^{D}(x, v) d t,
$$

and we call $G_{D}(x, v)$ the Green function for $D$. If $V$ is continuous on $\mathbb{R}^{d} \backslash\{0\}$, so that $V(x) \leq c|x|^{\alpha-d}$, then the strong Markov property yields, for $x, v \in D$,

$$
G_{D}(x, v)=V(x, v)-E^{x} V\left(X_{\tau_{D}}, v\right)=V(x, v)-\int_{D^{\mathrm{c}}} V(z, v) \omega_{D}^{x}(d z) .
$$


Here $V(x, v)=V(v-x)$. The Green function is symmetric: $G_{D}(x, v)=$ $G_{D}(v, x)$, continuous in $D \times D \backslash\{(x, v): x=v\}$, and it vanishes if $x \in D^{\mathrm{c}}$ or $v \in D^{\mathrm{c}}$.

Note that $V(x, v)$ is harmonic in $x$ on $\mathbb{R}^{d} \backslash\{v\}$. Indeed, if $x \in D$ and $\operatorname{dist}(D, v)>0$ then by $(23)$,

$$
V(x, v)=\int_{0}^{\infty} E^{x}\left[p\left(t-\tau_{D}, X_{\tau_{D}}, v\right) ; \tau_{D}<t\right] d t=E^{x} V\left(X_{\tau_{D}}, v\right) .
$$

Similarly, the Green function $v \mapsto G_{D}(x, v)$ is harmonic in $D \backslash\{x\}$.

By the Ikeda-Watanabe formula [34] we have

$$
\omega_{D}^{x}(A)=\int_{D} G_{D}(x, v) \nu(A-v) d v \quad \text { if } \operatorname{dist}(A, D)>0 .
$$

We note here that translation invariance of the Lebesgue measure and the Fubini-Tonelli theorem yield

$$
\iint \Phi(v) \Psi(v+z) m(d z) d v=\iint \Phi(v+z) \Psi(v) m(d z) d v
$$

for every symmetric measure $m$ and nonnegative functions $\Phi$ and $\Psi$. In particular, taking $m=\nu, \Phi(v)=G_{D}(x, v)$ and $\Psi(v)=\mathbf{1}_{A}(v)$ we get

$$
\int_{D} G_{D}(x, v) \nu(A-v) d v=\int_{A} \int_{-D+v} G_{D}(x, v-z) \nu(d z) d v .
$$

If the boundary of $D$ is smooth or even Lipschitz then

$$
\omega_{D}^{x}(\partial D)=0, \quad x \in D
$$

(see [50] and also [42], [54]). In this case $\omega_{D}^{x}$ is absolutely continuous with respect to the Lebesgue measure on $D^{\mathrm{c}}$. Its density function, or the Poisson kernel, is given by the formula

$$
P_{D}(x, y)=\int_{y-D} G_{D}(x, y-z) \nu(d z), \quad x \in D .
$$

Note that $D$ is then regular, because of (18) and scaling. In particular the above considerations apply to $D=B(0,1)$.

It follows from (9) that for every $r>0$ and $x \in \mathbb{R}^{d}$ the $P^{x}$ distribution of $\left\{X_{t}, t \geq 0\right\}$ is the same as the $P^{r x}$ distribution of $\left\{r^{-1} X_{r^{\alpha}} t, t \geq 0\right\}$. In particular,

$$
\omega_{D}^{x}(A)=\omega_{r D}^{r x}(r A) .
$$

We call (28) scaling, too. It shows that for $u$ harmonic on $D$, the dilation $u_{r}$ is harmonic on $r D$. A similar remark concerns translations.

By (26) we also obtain

$$
\int_{B(0,1 / 2)}|y|^{\alpha-d} \nu(A-y) d y=\int_{A} \int_{B(y, 1 / 2)}|y-z|^{\alpha-d} \nu(d z) d y, \quad A \subset \mathbb{R}^{d},
$$


and

$$
\int_{B(0,1 / 2)} \nu(A-y) d y=\int_{A} \nu(B(y, 1 / 2)) d y, \quad A \subset \mathbb{R}^{d} .
$$

Therefore we can express the relative Kato condition (RK) in an equivalent form:

$$
\int_{B(0,1 / 2)}|y|^{\alpha-d} \nu(A-y) d y \leq K \int_{B(0,1 / 2)} \nu(A-y) d y, \quad A \subset \mathbb{R}^{d} .
$$

We remark that (RK) is a local condition at infinity: the inequality in (2) only needs to be verified for large $y \in \mathbb{R}^{d}$. In particular, if it holds for $|y|>1$ then it holds for all $y \in \mathbb{R}^{d}$, possibly with a different constant (see [17]). Note that the reverse of (2) (and (29)) always holds, so actually (RK) means comparability of both sides of (2) (and (29)).

In what follows we let $G=G_{B(0,1)}, P=P_{B(0,1)}$ and we define

$$
s(x)=E^{x} \tau_{B(0,1)}=\int_{B(0,1)} G(x, v) d v .
$$

Explicit formulas for these functions for $\nu(d y)=|y|^{-d-\alpha} d y$ are known and may give some insight into the general situation. They are essentially due to M. Riesz (see, e.g., [15], [11], [40], [9], [28]). In particular (for isotropic $\nu$ ) we have

$$
P(x, y)=C_{\alpha}^{d}\left[\frac{1-|x|^{2}}{|y|^{2}-1}\right]^{\alpha / 2}|x-y|^{-d}, \quad|x|<1,|y|>1 .
$$

The following two lemmas are consequences of symmetry and nondegeneracy of the spectral measure $\mu$. They can be proved similarly to Lemmas 4 and 10 of [17], so we skip the proofs.

Lemma 10. There exist $\varepsilon=\varepsilon(\alpha, \mu) \in(0,1)$ and $C=C(\alpha, \mu)$ such that

$$
\nu(B(x, 1-\varepsilon)) \geq C, \quad 1-\varepsilon<|x|<1 .
$$

Lemma 11. There exists $C=C(\alpha, \mu)$ such that

$$
s(x) \leq C\left(1-|x|^{2}\right)^{\alpha / 2}, \quad|x|<1 .
$$

For clarity we make the following remark on nondegeneracy of $\nu$. If a symmetric Lévy measure $\nu$ is concentrated on a proper linear subspace $M$ of $\mathbb{R}^{d}$, and a function $u$ is constant on $x+M$ for every $x \in \mathbb{R}^{d}$, then $u$ is harmonic on $\mathbb{R}^{d}$ (relative to $\nu$ ). However, such functions in general violate the Harnack inequality. Thus our standing assumption of the nondegeneracy of $\nu$ is a necessary, or nonrestrictive, condition for Harnack's inequality in Theorem 3. 
5. Necessity of the relative Kato condition. In this short section we assume that Harnack's inequality (1) holds. We make no further assumptions on $\nu$ beyond those in Section 2. In particular our considerations do not depend on the estimates in Section 3.

Lemma 12. Harnack's inequality implies the relative Kato condition.

Proof. We first consider the case $d>\alpha$. We claim that

$$
V(x) \approx|x|^{\alpha-d}, \quad x \in \mathbb{R}^{d} .
$$

Indeed, for all $|x|=1, \mathbb{V}(B(x, 1 / 4))=\int_{B(x, 1 / 4)} V(v) d v \leq \mathbb{V}(B(0,2))<\infty$, so there exists $v \in B(x, 1 / 4)$ such that $V(v) \leq \mathbb{V}(B(0,2)) /|B(0,1 / 4)|$. By Harnack's inequality $V(x) \leq c V(v)$. The estimate (32) follows from (12) and (19).

Let $g(v)=\min (G(0, v), 1)$. We claim that

$$
G(x, v) \approx g(v)|v-x|^{\alpha-d} \quad \text { if }|x|<1 / 2 \text { and }|v|<1 .
$$

Indeed, by (32) and (24) for small $\delta>0$ we have

$$
G(x, v) \approx|v-x|^{\alpha-d}, \quad|x|<1 / 2,|x-v|<\delta .
$$

Harnack's inequality implies that $G(x, v) \approx|v-x|^{\alpha-d}$ provided $|x|<1 / 2$ and $|v|<3 / 4$, and also $G(x, v) \approx G(0, v)$ if $|x|<1 / 2$ and $|v|>3 / 4$. Note that $g$ is locally bounded from below on $B(0,1)$. This completes the proof of (33).

For every $A \subset \mathbb{R}^{d}$ the function $x \mapsto \omega_{B(0,1)}^{x}(A)$ is nonnegative on $\mathbb{R}^{d}$ and regular harmonic in $B(0,1)$. Harnack's inequality (1), (25), (33), and Fubini-Tonelli yield

$$
\begin{aligned}
\omega_{B(0,1)}^{0}(A) & \approx \int_{B(0,1 / 2)} \omega_{B(0,1)}^{x}(A) d x \approx \int_{B} \int_{B(0,1 / 2)} g(v)|v-x|^{\alpha-d} \nu(A-v) d v d x \\
& \approx \int_{B} g(v) \nu(A-v) d v .
\end{aligned}
$$

This and (25) yield

$$
\int_{B} g(v)|v|^{\alpha-d} \nu(A-v) d v \approx \int_{B} g(v) \nu(A-v) d v .
$$

To this "approximate equality" we add the following one:

$$
\int_{B \backslash B(0,3 / 4)}|v|^{\alpha-d} \nu(A-v) d v \approx \int_{B \backslash B(0,3 / 4)} \nu(A-v) d v,
$$

and we obtain

$$
\int_{B}|v|^{\alpha-d} \nu(A-v) d v \approx \int_{B} \nu(A-v) d v, \quad A \subset B^{\mathrm{c}} .
$$

The change of variable $v=2 u$ yields (29) and (2). 
In the case $d \leq \alpha$ we have $d=1$, and so $\nu(d y)=c|y|^{-1-\alpha} d y$, which satisfies (RK).

6. Sufficiency of the relative Kato condition. In what follows we assume that (RK) holds for $\nu$. We will also assume that $d>\alpha$ unless stated otherwise.

The key step in the proof of Harnack's inequality is the following estimate for the Green function of the ball, which we prove after a sequence of lemmas. We note that it is essentially the same as (33), but proved under explicit assumptions on $\nu$ rather than by stipulating Harnack's inequality. The estimate was suggested by the sharp estimates of the Green function of Lipschitz domains [38] for isotropic $\nu$ (see also [13]). We also refer the reader to [39, 21] for more explicit estimates for smooth domains and to, e.g., [15] for explicit formulas for the ball in the case of isotropic $\nu$.

Proposition 1. $G(x, v) \approx s(v)|v-x|^{\alpha-d}$ provided $|x|<1 / 2$ and $|v|<1$.

Lemma 13. $\nu$ is a $(d-\alpha)$-measure on $\mathbb{S}$.

Proof. Indeed, for $|x|=1,0<r<1 / 2$ by (2) we obtain

$$
\nu(B(x, r)) \leq r^{d-\alpha} \int_{B(x, 1 / 2)}|x-z|^{\alpha-d} \nu(d z) \leq K \nu\left(B(0,1 / 2)^{c}\right) r^{d-\alpha} .
$$

Theorem 1 implies that $V$ is continuous on $\mathbb{R}^{d} \backslash\{0\}$. Consequently, $V(x) \approx$ $|x|^{\alpha-d}$ and $G(x, y)$ is continuous on $B \times B \backslash\{(x, y): x=y\}$.

Lemma 14. $G(x, v) \approx|v-x|^{\alpha-d}$ if $|x|<1 / 2,|v|<3 / 4$.

We skip the proof as it is the same as the one of Lemma 6 in [17].

We note that $\lim _{x \rightarrow z} G(x, v)=0$ for all $v \in B(0,1)$ and $z \in \mathbb{S}$ because the measures $\omega_{B(0,1)}^{x}$ weakly converge to $\delta_{z}$. This is related to the regularity of $B(0,1)$, and follows, e.g., from the estimate

$$
\omega_{B(x, 1-|x|)}^{x}\left(B(0,1)^{\mathrm{c}}\right) \geq c,
$$

which is a consequence of scaling, nondegeneracy of $\nu$ (compare (31)), and (25).

We will employ the operator

$$
\mathcal{U}_{r} \phi(x)=\frac{E^{x} \phi\left(X_{\tau_{B(x, r)}}\right)-\phi(x)}{E^{x} \tau_{B(x, r)}},
$$

whenever the expression is well defined for given $\phi, r>0$ and $x$. We note that $\mathcal{U}_{r}$ is implicitly used in [7, Chapter III, §17]. Clearly, if $h$ is harmonic in $D, x \in D$, and $r<\operatorname{dist}\left(x, D^{\mathrm{c}}\right)$, then $\mathcal{U}_{r} h(x)=0$. We note that

$$
\mathcal{U} \phi(x)=\lim _{r \downarrow 0} \mathcal{U}_{r} \phi(x)
$$

is the Dynkin characteristic operator, which was used in [17] in a similar way. 
We record the following observation (maximum principle).

LEMma 15. If there is $r>0$ such that $\mathcal{U}_{r} h(x)>0$ then

$$
h(x)<\sup _{y \in \mathbb{R}^{d}} h(y) .
$$

Lemma 16. There exists $C=C(\alpha, \mu)$ such that

$$
G(x, v)<C s(v), \quad|x|<1 / 2,3 / 4<|v|<1 .
$$

Proof. By the strong Markov property we have

$$
\begin{aligned}
s(v) & =E^{v} \tau_{B}=E^{v}\left(\tau_{A}+\tau_{B(0,1)} \circ \theta_{\tau_{A}}\right)=E^{v} \tau_{A}+E^{v} E^{X_{\tau_{A}}} \tau_{B(0,1)} \\
& =E^{v} \tau_{A}+E^{v} s\left(X_{\tau_{A}}\right), \quad v \in \mathbb{R}^{d}, A \subset B(0,1),
\end{aligned}
$$

which yields $\mathcal{U}_{r} s(v)=-1$ for $v \in B(0,1)$ and $r<1-|v|$.

For $n \in\{1,2, \ldots\}$ and $x \in B(0,1 / 2)$ we let $g(v)=G(x, v)$ and $g_{n}(v)=$ $\min (G(x, v), n)$. For $v \in B(x, 1 / 8)^{\mathrm{c}}$ we have $G(x, v) \leq c_{1}|x-v|^{\alpha-d}$, hence $g_{n}(v)=G(x, v)$ provided $n \geq c_{1} 8^{d-\alpha}$. By harmonicity of $g$ on $B(0,1) \backslash\{x\}$, scaling property, (25) and (2) we find that for $v \in B(0,1) \backslash B(0,3 / 4)$ and $r<\min (1-|v|, 1 / 16)$,

$$
\begin{aligned}
\mathcal{U}_{r} g_{n}(v) & =\mathcal{U}_{r}\left(g_{n}-g\right)(v) \\
& =\frac{1}{E^{0} \tau_{B(0,1)}} \int_{B(0,1)} G(0, w) \int\left(g_{n}-g\right)(v+r w+z) \nu(d z) d w \\
& \geq \frac{-c_{2}}{s(0)} \int_{B(0,1)} G(0, w) \int_{B(x-v-r w, 1 / 8)}|x-v-r w-z|^{\alpha-d} \nu(d z) d w \\
& \geq \frac{-c_{2} K}{s(0)} \int_{B(0,1)} G(0, w) \nu(B(x-v-r w, 1 / 8)) d w \geq-c_{3} .
\end{aligned}
$$

If $a>c_{3}$ then

$$
\mathcal{U}_{r}\left(a s-g_{n}\right)(v)=-a-\mathcal{U}_{r} g_{n}(v) \leq-a+c_{3}<0 .
$$

By scaling

$$
\begin{aligned}
s(v) & \geq E^{v} \tau_{B(v, 1-|v|)}=(1-|v|)^{\alpha} E^{0} \tau_{B(0,1)} \\
& \geq 4^{-\alpha} E^{0} \tau_{B(0,1)}, \quad|v|<3 / 4 .
\end{aligned}
$$

Since $g_{n}(v) \leq n$, we see that $a s(v)-g_{n}(v)>0$ for $v \in B(0,3 / 4)$ provided $a>n /\left(4^{-\alpha} E^{0} \tau_{B(0,1)}\right)$.

Let $a_{0}=\max \left[c_{3}, n /\left(4^{-\alpha} E^{0} \tau_{B(0,1)}\right)\right]+1$ and $h(v)=a_{0} s(v)-g_{n}(v)$. We have $h(v) \geq 0$ for $v \in \overline{B(0,3 / 4)}, h(v)=0$ for $v \in B(0,1)^{\mathrm{c}}$ and $\mathcal{U}_{r} h(v)<0$ for $v \in B(0,1) \backslash B(0,3 / 4), r<\min (1-|v|, 1 / 16)$. Lemma 15 and continuity of $h$ yield $h(v) \geq 0$ in $B(0,1)$. Since $g_{n}=g$ on $B(0,3 / 4)^{\mathrm{c}}$, the lemma follows. 
Lemmas 16 and 11 yield the following conclusion:

$$
G(x, v) \leq C(1-|v|)^{\alpha / 2}, \quad|x|<1 / 2,3 / 4<|v|<1 .
$$

Lemma 17. There is $C=C(\alpha, \mu)$ such that $G(x, v) \geq C s(v)$ provided $|x|<1 / 2$ and $|v|<1$.

Proof. Let $x \in B(0,1 / 2)$. We fix $\varepsilon$ such that (31) is satisfied. Lemma 14 yields $G(x, v) \geq c_{1}>0$ for $v \in B(0,1-\varepsilon)$. Let $n \in\{1,2, \ldots\}$ be such that $c_{1} \geq 2 / n$. By (35) there is $\eta>0$ such that $G(x, v) \leq 1 / n$ for $v \in$ $B(0,1) \backslash B(0,1-\eta)$. Let $g(v)=G(x, v)$ and $g_{n}(v)=\min (g(v), 1 / n)$. We have

$$
g_{n}(v)=g(v), \quad v \in B(0,1) \backslash B(0,1-\eta),
$$

and

$$
g(v)-g_{n}(v) \geq 2 / n-1 / n=1 / n, \quad v \in B(0,1-\varepsilon),
$$

hence by Lemma 10 for $v \in B(0,1) \backslash \overline{B(0,1-\eta)}$ and $r<\min (1-|v|$, $(\varepsilon-\eta) / 2)$ we obtain

$$
\begin{aligned}
\mathcal{U}_{r} g_{n}(v) & =\mathcal{U}_{r}\left(g_{n}-g\right)(v) \\
& =\frac{1}{s(0)} \int_{B(0,1)} G_{B(0,1)}(0, w) \int\left(g_{n}-g\right)(v+r w+z) \nu(d z) d w \\
& \leq-\frac{1}{n} \frac{1}{s(0)} \int_{B(0,1)} G_{B(0,1)}(0, w) \nu(B(v+r w, 1-\varepsilon)) d w \leq-\frac{c_{2}}{n} .
\end{aligned}
$$

For $a>0$ we have

$$
\mathcal{U}_{r}\left(a g_{n}-s\right)(v) \leq-c_{2} a / n+1, \quad v \in B(0,1) \backslash B(0,1-\eta) .
$$

This is negative if $a>n / c_{2}$. Furthermore $s(v) \leq c_{3}$ for $v \in B(0,1)$ and $g_{n}(v) \geq c_{4}>0$ for $v \in B(0,1-\eta)$. Thus $a g_{n}(v)-s(v) \geq a c_{4}-c_{3}>0$ for $v \in B(0,1-\eta)$ if only $a>c_{3} / c_{4}$. Note that our estimates do not depend on $x$, provided $|x|<1 / 2$. Let $a_{0}=\max \left(c_{3} / c_{4}, n / c_{2}\right)+1$ and $h(v)=$ $a_{0} g_{n}(v)-s(v)$. We have $h(v) \geq 0$ for $v \in \overline{B(0,1-\eta)}$, and $\mathcal{U}_{r} h(v)<0$ for $v \in B(0,1) \backslash B(0,1-\eta)$. By Lemma 15 and the continuity of $h$ we get $h(v) \geq 0$ in $B(0,1)$, and the lemma follows.

Proof of Proposition 1. The estimate is a consequence of (34), Lemma 14, 16 , and 17.

Maciej Lewandowski [41] has informed us that he recently proved the converse of the inequality in Lemma 11 . This implies

$$
G(x, v) \approx\left(1-|v|^{2}\right)^{\alpha / 2}|v-x|^{\alpha-d}, \quad|x|<1 / 2,|v|<1 .
$$

We will not use (36) below; the less explicit estimate in Lemma 17 suffices for our purposes. Note that the asymptotics of $G$ at the pole is different when $d=1 \leq \alpha$ (see, e.g., [15]). 
Lemma 18. (RK) implies Harnack's inequality for all $d \in\{1,2, \ldots\}$ and $\alpha \in(0,2)$.

Proof. By translation and scaling invariance of the class of harmonic functions and by a covering argument we only need to verify that

$$
u(0) \leq c u(x), \quad|x|<1 / 2,
$$

whenever $u$ is nonnegative on $\mathbb{R}^{d}$ and regular harmonic on $B(0,1)$. For this to hold it is sufficient to have, with the same constant $c$,

$$
P(0, y) \leq c P(x, y), \quad|x|<1 / 2,|y|>1 .
$$

If $d=1$, then (37) follows from (30). Thus we only need to examine the case $d>\alpha$. By the decomposition $B(0,1)=B(0,1 / 2) \cup[B(0,1) \backslash B(0,1 / 2)]$, (27), Proposition $1,(2)$, and the fact that $s$ is bounded away from zero on compact subsets of $B(0,1)$ (cf. (34)), we obtain

$$
\begin{aligned}
P(0, y) & \approx \int_{B(y, 1)} s(y-v)|y-v|^{\alpha-d} \nu(d v) \approx \int_{B(y, 1)} s(y-v) \nu(d v) \\
& \leq c \int_{B(y, 1)} s(y-v)|y-v-x|^{\alpha-d} \nu(d v) \\
& \approx P(x, y), \quad|x|<1 / 2,|y|>1 .
\end{aligned}
$$

\section{Proof of Theorem 3. See Lemmas 12 and 18.}

We conclude with a few remarks and open problems.

By translation and dilation invariance of the class of harmonic functions considered, and by a covering argument, Harnack's inequality holds for every compact subset of every connected domain of harmonicity. We note that: (1) it does not generally hold for disconnected open sets, as the support of $y \mapsto P(x, y)$ may be smaller than $B(0,1)^{\mathrm{c}}$ (see $(25)$ ), (2) it does hold for all open sets if $\nu$ is isotropic (this follows from (30), or see [14]).

We consider the following examples of measures $\nu$. (RK) holds for $\nu_{1}(d y)$ $\approx|y|^{-d-\alpha} d y$ (both sides of (2) may be explicitly estimated). Next, let $\xi \in \mathbb{S}$, $0<r<\sqrt{2}$, and $C=\mathbb{S} \cap[B(\xi, r) \cup B(-\xi, r)]$. Then (RK) holds for $\nu_{2}(d y)=$ $\mathbf{1}_{C}(y /|y|)|y|^{-d-\alpha} d y$ (see [17]).

On the other hand, consider balls $B_{n} \subset B_{n}^{\prime}$ centered at $\mathbb{S}$, with radii $4^{-n}$ and $2^{-n}$, respectively, and such that $\left\{B_{n}^{\prime}\right\}$ are pairwise disjoint. Let $C=\bigcup_{n \geq n_{0}} B_{n}$ and let $\nu_{3}(d y)=\mathbf{1}_{C}(y /|y|)|y|^{-d-\alpha} d y$. If $d-1>\alpha$ then (RK) does not hold for $\nu_{3}$ ([17]) even though $\nu_{3}$ is bounded by $\nu_{1}$.

Let $B_{\xi, r}=B(\xi, r) \cap \mathbb{S}$. By integrating in polar coordinates we can give the characterization of the relative Kato condition in terms of the spectral measure $\mu$ and $B_{\xi, r}$ (cf. [17]). Let $d-\alpha>1$. Then (RK) holds for $\nu$ if 
and only if

$$
\int_{B_{\xi, r}}(|\eta-\xi| / r)^{\alpha-(d-1)} \mu(d \eta) \leq c \mu\left(B_{\xi, r}\right), \quad \xi \in \mathbb{S}, 0<r<c .
$$

In the case $d=2, \alpha=1,(\mathrm{RK})$ is equivalent to

$$
\int_{B_{\xi, r}} \log (2 r /|\eta-\xi|) \mu(d \eta) \leq c \mu\left(B_{\xi, r}\right), \quad \xi \in \mathbb{S}, 0<r<c .
$$

In the case of $d=2$ and $\alpha>1$, (RK) is always satisfied. We omit the proofs.

Corollary 19. If $d-1<\alpha$ then Harnack's inequality holds for $\mathcal{A}$.

This may be extended as follows. We will say that $\nu$ is a strict $\gamma$-measure if

$$
\nu(B(x, r)) \approx r^{\gamma} \quad \text { provided } x \in \operatorname{supp} \nu,|x|=1,0<r<1 / 2
$$

(cf. (13)). Of course, if $\nu$ is a (strict) $\gamma$-measure on $\mathbb{S}$ then $\mu$ is a (strict) $(\gamma-1)$ measure (on $\mathbb{S}$ ). This observation and (38) yield the following conclusion, which we state without proof.

COROLlary 20. If $\nu$ is a strict $\gamma$-measure with $\gamma>d-\alpha$, then Harnack's inequality holds for $\mathcal{A}$.

The example of $\nu_{3}$ shows the importance of the strictness assumption. We interpret $(\mathrm{RK})$ as a property of balance or firmness of $\nu$. As such it is close to the reverse Hölder condition with exponent $q>d / \alpha$ (see [17]).

If $\mu(\xi)>0$ for some $\xi \in \mathbb{S}$ then $\nu$ is a 1-measure only. By Theorem 2 the potential kernel $V$ is unbounded on $\mathbb{S}$ if $1>d-2 \alpha$ (in fact, if $1 \geq d-2 \alpha$, see [53, Theorem 1.1], [17]). That $V$ may be infinite on rays emanating from the origin shows that harmonic functions cannot be defined pointwise by means of $\mathcal{A}$. In general they even lack finiteness in the domain of harmonicity (but see [14] and [44] in this connection). Thus the potential-theoretic properties of the operators $\mathcal{A}$ are very diverse for the measures $\nu$ considered here. This is in sharp contrast with the fact that the exponents $\Phi$ (see (5)) are all comparable and the same is true of the corresponding Dirichlet forms ([27], see also [25]). The boundary potential theory of $\mathcal{A}$ will generally be very different from that of the fractional Laplacian (see [51, p. 199] for a simple remark on this subject).

We mention a number of interesting topics deserving further study: (1) characterization of continuity and higher order regularity of $V$ on $\mathbb{S}([6])$, (2) the boundary Harnack principle (cf. $[12,16,51])$, the corresponding approximate factorization of $G(x, v)$ for all $x, v \in B(0,1)$ (cf. [13, 31, 20] and Proposition 1 above), and related boundary problems (cf. [1, 19]), (3) study of other Lévy measures which are products in polar coordinates, (4) study of similar nonlocal operators $\mathcal{A}$ which are not translation invariant $([2,47])$. 


\section{References}

[1] R. Bañuelos and K. Bogdan, Symmetric stable processes in cones, Potential Anal. 21 (2004), 263-288.

[2] M. Barlow, R. F. Bass and M. Kassmann, preprint, 2005.

[3] R. F. Bass, Diffusions and Elliptic Operators, Springer, New York, 1998.

[4] R. F. Bass, Stochastic differential equations with jumps, Probab. Surv. 1 (2004), 1-19 (electronic).

[5] R. F. Bass and M. Kassmann, Harnack inequalities for non-local operators of variable order, Trans. Amer. Math. Soc. 357 (2005), 837-850.

[6] R. F. Bass and D. A. Levin, Harnack inequalities for jump processes, Potential Anal. 17 (2002), 375-388.

[7] C. Berg and G. Forst, Potential Theory on Locally Compact Abelian Groups, Springer, 1975.

[8] J. Bertoin, Lévy Processes, Cambridge Univ. Press, Cambridge, 1996.

[9] J. Bliedtner and W. Hansen, Potential Theory. An Analytic and Probabilistic Approach to Balayage, Springer, Berlin, 1986.

[10] R. M. Blumenthal and R. K. Getoor, Markov Processes and Potential Theory, Academic Press, New York, 1968.

[11] R. M. Blumenthal, R. K. Getoor, and D. B. Ray, On the distribution of first hits for the symmetric stable processes, Trans. Amer. Math. Soc. 99 (1961), 540-554.

[12] K. Bogdan, The boundary Harnack principle for the fractional Laplacian, Studia Math. 123 (1997), 43-80.

[13] - Sharp estimates for the Green function in Lipschitz domains, J. Math. Anal. Appl. 243 (2000), 326-337.

[14] K. Bogdan and T. Byczkowski, Potential theory for the $\alpha$-stable Schrödinger operator on bounded Lipschitz domains, Studia Math. 133 (1999), 53-92.

[15] - - - Potential theory of the Schrödinger operator based on the fractional Laplacian, Probab. Math. Statist. 20 (2000), 293-335.

[16] K. Bogdan, A. Stós and P. Sztonyk, Potential theory for Lévy stable processes, Bull. Polish Acad. Sci. Math. 50 (2002), 361-372.

[17] K. Bogdan and P. Sztonyk, Harnack's inequality for stable Lévy processes, Potential Anal. 22 (2005), 133-150.

[18] K. Bogdan and T. Żak, On Kelvin transformation, J. Theoret. Probab. 19 (2006), $89-120$.

[19] K. Burdzy and T. Kulczycki, Stable processes have thorns, Ann. Probab. 31 (2003), 170-194.

[20] Z.-Q. Chen and P. Kim, Green function estimate for censored stable processes, Probab. Theory Related Fields 124 (2002), 595-610.

[21] Z.-Q. Chen and R. Song, Martin boundary and integral representation for harmonic functions of symmetric stable processes, J. Funct. Anal. 159 (1998), 267-294.

[22] K. L. Chung, Lectures from Markov Processes to Brownian Motion, Springer, New York, 1982.

[23] -, Doubly-Feller process with multiplicative functional, in: Seminar on Stochastic Processes (Gainesville, FL, 1985), Progr. Probab. Statist. 12, Birkhäuser, Boston, MA, 1986, 63-78.

[24] K. L. Chung and Z. Zhao, From Brownian Motion to Schrödinger's Equation, Springer, New York, 1995.

[25] B. Dyda, On comparability of integral forms, J. Math. Anal. Appl. 318 (2006), $564-577$. 
[26] J. Dziubański, Asymptotic behaviour of densities of stable semigroups of measures, Probab. Theory Related Fields 87 (1991), 459-467.

[27] M. Fukushima, Y. Ōshima, and M. Takeda, Dirichlet Forms and Symmetric Markov Processes, de Gruyter, Berlin, 1994.

[28] R. K. Getoor, First passage times for symmetric stable processes in space, Trans. Amer. Math. Soc. 101 (1961), 75-90.

[29] D. Gilbarg and N. S. Trudinger, Elliptic Partial Differential Equations of Second Order, Springer, Berlin, 2001.

[30] P. Głowacki and W. Hebisch, Pointwise estimates for densities of stable semigroups of measures, Studia Math. 104 (1993), 243-258.

[31] W. Hansen, Uniform boundary Harnack principle and generalized triangle property, J. Funct. Anal. 226 (2005), 452-484.

[32] S. Hiraba, Asymptotic estimates for densities of multi-dimensional stable distributions, Tsukuba J. Math. 27 (2003), 261-287.

[33] W. Hoh, Pseudo differential operators generating Markov processes, Habilitationsschrift, Univ. Bielefeld, 1998.

[34] N. Ikeda and S. Watanabe, On some relations between the harmonic measure and the Lévy measure for a certain class of Markov processes, J. Math. Kyoto Univ. 2 (1962), 79-95.

[35] N. Jacob, Pseudo Differential Operators and Markov Processes, Vol. I: Fourier Analysis and Semigroups, Imperial College Press, London, 2001.

[36] —, Pseudo-Differential Operators and Markov Processes, Vol. II: Generators and Their Potential Theory, Imperial College Press, London, 2002.

[37] N. Jacob and R. Schilling, Lévy-type processes and pseudodifferential operators, in: Lévy Processes. Theory and Applications, Birkhäuser, Boston, MA, 2001, 139-168.

[38] T. Jakubowski, The estimates for the Green function in Lipschitz domains for the symmetric stable processes, Probab. Math. Statist. 22 (2002), 419-441.

[39] T. Kulczycki, Properties of Green function of symmetric stable processes, ibid. 17 (1997), 339-364.

[40] N. S. Landkof, Foundations of Modern Potential Theory, Springer, New York, 1972.

[41] M. Lewandowski, private communication 2005.

[42] P. W. Millar, First passage distributions of processes with independent increments, Ann. Probab. 3 (1975), 215-233.

[43] J. Picard, Density in small time at accessible points for jump processes, Stochastic Process. Appl. 67 (1997), 251-279.

[44] J. Picard and C. Savona, Smoothness of harmonic functions for processes with jumps, ibid. 87 (2000), 69-91.

[45] M. Rao, R. Song and Z. Vondraček, Green function estimates and Harnack inequality for subordinate Brownian motions, Potential Anal. 25 (2006), 1-27.

[46] K.-I. Sato, Lévy Processes and Infinitely Divisible Distributions, Cambridge Univ. Press, 1999.

[47] R. Song and Z. Vondraček, Harnack inequality for some classes of Markov processes, Math. Z. 246 (2004), 177-202.

[48] D. W. Stroock, Diffusion processes associated with Lévy generators, Z. Wahrsch. Verw. Gebiete 32 (1975), 209-244.

[49] -, Markov Processes from K. Itô's Perspective, Ann. of Math. Stud. 155, Princeton Univ. Press, Princeton, NJ, 2003.

[50] P. Sztonyk, On harmonic measures for Lévy processes, Probab. Math. Statist. 20 (2000), 383-390.

[51] —, Boundary potential theory for stable processes, Colloq. Math. 95 (2003), 191-206. 
[52] S. J. Taylor, Sample path properties of a transient stable process, J. Math. Mech. 16 (1967), 1229-1246.

[53] T. Watanabe, Asymptotic estimates of multi-dimensional stable densities and their applications, Trans. Amer. Math. Soc. 359 (2007), 2851-2879.

[54] J.-M. Wu, Harmonic measures for symmetric stable processes, Studia Math. 149 (2002), 281-293.

K. Bogdan

Institute of Mathematics

Polish Academy of Sciences

and

Institute of Mathematics

Wrocław University of Technology

Wybrzeże Wyspiańskiego 27

50-370 Wrocław, Poland

E-mail: bogdan@pwr.wroc.pl
P. Sztonyk

Institute of Mathematics Wrocław University of Technology Wybrzeże Wyspiańskiego 27 50-370 Wrocław, Poland E-mail: sztonyk@im.pwr.wroc.pl

Received September 5, 2005

Revised version May 30, 2007 